\title{
STATE-TO-STATE DYNAMICS OF MOLECULAR ENERGY TRANSFER
}

\author{
Final Performance Report \\ For Period
}

April 1, 1989 to March 31, 1992

W. Ronald Gentry

University of Minnesota

Minneapolis, Minnesota 55455

Oct. 1, 1991

Prepared for

The U.S. Department of Energy

under Grant No. DE-FG02-86ER13515

\begin{abstract}
NOTICE
This report was prepared as an acccunt of work sponsored by the United States Government. Neither the United States nor the United States Department of Energy, nor any of their employees, nor any of their contractors, subcontractors, or their employees, makes any warranty, express or implied, or assumes any legal liability or responsibility for the accuracy, completeness, or usefulness of any information, apparatus, product or process disclosed or represents that its use would not infringe on privately owned rights.
\end{abstract}

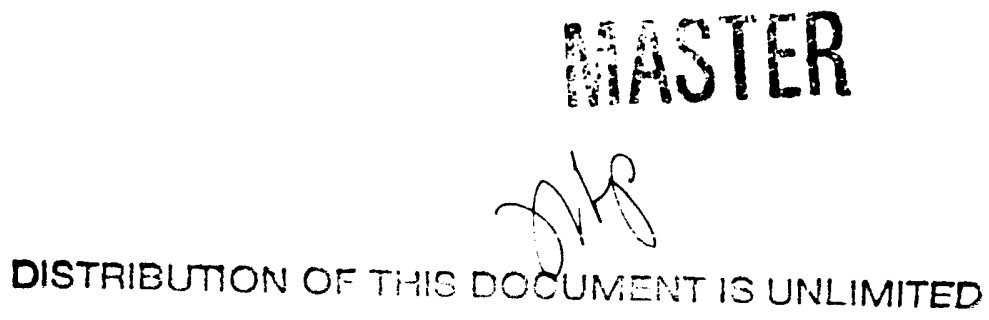


Our experimental results under this DOE project to date encompass studies of: (1) state- and mode-selective vibrational excitation of $\mathrm{I}_{2}$, aniline, $p-$ difluorobenzene (pDFB) and glyoxal in collisions with various species, (2) rotationally-resolved inelastic scattering in $\mathrm{I}_{2}+\mathrm{He}, \mathrm{pDFB}+\mathrm{He}$, and glyoxal $+\mathrm{He}$ collisions, and (3) state-to-state vibrational energy transfer from highly-excited initial vibrational states of $\mathrm{I}_{2}$ in collisions with He. The following papers on work completed under this DOE grant have either been published or are being prepared for publication.

1. G. Hall, K. Liu, M. J. McAuliffe, C. F. Giese and W. R. Gentry, "Pulsed Molecular Beam Study of State-to-State Vibrational Excitation in the $\mathrm{He}+\mathrm{I}_{2}$ Collisions--Energy Dependence of the $\mathrm{v}=0 \rightarrow 1$ Cross Section, J. Chem. Phys. 78, 5260 (1983).

2. K. Liu, G. Hall, M. J. McAuliffe, C. F. Giese and W. R. Gentry, "Kinetic Energy Dependence of Cross Sections for State-to-State Vibrational Excitation in Collisions of $\mathrm{S}_{0}$ Aniline with He," J. Chem. Phys. 80, 3494 (1984).

3. G. Hall, K. Liu, M. J. McAuliffe, C. F. Giese and W. R. Gentry, "State-to-State Vibrational Excitation of $\mathrm{I}_{2}$ in Collisions with He," J. Chem. Phys. 81, 5577 (1984).

4. G. Hall, "Vibrational Excitation in Molecular Collisions," Ph.D. Thesis, University of Minnesota (1985).

5. W. R. Gentry, "Pulsed Molecular Beam Experiments," Proc. 14th International Symposium on Rarified Gas Dynamics (University of Tokyo Press, 1984).

6. G. Hall, C. F. Giese and W. R. Gentry, "Vibrational Excitation of Ground-State p-Difluorobenzene in Collisions with He," J. Chem. Phys. 83, 5343 (1985).

7. W. R. Gentry, "State-to-State Energy Transfer in Collisions of Neutral Molecules," in Electronic and Atomic Collisions, ed. by D. C. Lorents, W. E. Meyerhof and J. R. Peterson (Elsevier, Amsterdam, 1986), pp. 13-22.

8. G. Hall, K. Liu, M. J. McAuliffe, C. F. Giese and W. R. Gentry, "State-to-State Vibrational Excitation of $\mathrm{I}_{2}$ in Collisions with $\mathrm{H}_{2}$ and $\mathrm{D}_{2}, "$ J. Chem. Phys. 84, 2624 (1986).

9. W. R. Gentry, "Low-Energy Pulsed Beam Sources," Ch. 3 in Atomic and Molecular Beam Methods, ed. by G. Scoles (Oxford University Press, 1988). 
10. V. A. Shamamian, "State-Resolved Rotational Excitation Dynamics of Iodine and para-Difluorobenzene in Collisions with He," Ph.D. Thesis, University of Minnesota (1989).

11. Z. Ma, S. D. Jons, C. F. Giese and W. R. Gentry, "Crossed Beam Studies of State-toState Vibrational Energy Transfer from the $v=5$ Excited State of $\mathrm{I}_{2}\left({ }^{1} \Sigma_{\mathrm{g}}{ }^{+}\right)$Prepared by Stimulated Emission Pumping," J. Chem. Phys. 94, 8608 (1991).

12. Z. Ma, "Crossed Beam Studies of State-to-State Energy Transfer in Glyoxal and Iodine," Ph.D. Thesis, University of Minnesota (1991).

13. V. A. Shamamian, D. L. Catlett, C. F. Giese and W. R. Gentry, "State-Resolved Rotationally Inelastic Scattering in Collisions of $\mathrm{I}_{2}\left(\mathrm{X}^{1} \Sigma_{\mathrm{g}}{ }^{+}, \mathrm{v}=0\right)$ with $\mathrm{He},{ }^{n}$ in preparation.

14. V. A. Shamamian, D. L. Catlett, Z. Ma, S. A. Jons, C. F. Giese and W. R. Gentry, "Rotational Excitation Dynamics in Collisions of p-Diffuorobenzene with He," in preparation.

15. Z. Ma, S. A. Jons, C. F. Giese and W. R. Gentry, "Crossed Beam Studies of Energy Transfer in $S_{o}$ trans-Glyoxal + He Collisions: I. Vibrational Excitation and $k$ Resolved Rotational Excitation," in preparation.

16. S. A. Jons, Z. Ma, C. F. Giese and W. R. Gentry, "Crossed Beam Studies of Energy Transfer in $\mathrm{S}_{\mathrm{o}}$ trans-Glyoxal $+\mathrm{He}$ Collisions: II. Experiments on Fully-Resolved $j, k$ Rotational Transitions," in preparation.

17. Z. Ma, S. A. Jons, C. F. Giese and W. R. Gentry, "Quantum Number and Kinetic Energy Dependence of Cross Sections for State-to-State Vibrational Energy Transfer from the Excited States of $\mathrm{I}_{2}\left({ }^{1} \Sigma_{\mathrm{g}}{ }^{+}\right)$in Collisions with He, in preparation.

The large backlog of articles to be published on this work is a consequence of several factors. The most important of these was the necessity of moving the apparatus to a new laboratory to make possible the incorporation of stimulated emission pumping of the primary beam into the experiments. The previous laboratory could not accommodate the additional laser system needed for SEP, which consists of two Lambda Physik 3002E dye lasers synchronously pumped by a Lambda Physik LPX-205i excimer laser. At the same time the molecular beam apparatus was moved, the data acquisition system was completely rebuilt to improve data handling and allow for control of the additional lasers. Below, we summarize briefly the results not yet published. 


\section{A. Energy Transier from Vibrationally Excited States of the Ground Electronic State in $I_{2}+$ He Collisions}

Our most significant accomplishment during the past grant period has been the first-ever measurements of cross sections for state-to-state vibrational transitions of molecules in the ground electronic state, as a function of both kinetic energy and the initial vibrational quantum number. These are also the first crossed beam scattering experiments anywhere to incorporate the preparation of an initial excited state by stimulated emission pumping. We have been working for several years toward this goal, beginning with the acquisition of the SEP laser system through the DOE University Research Instrumentation Program. These experiments are certainly the most technically challenging which we have faced to date, incorporating as they do the two pulsed molecular beams, a total of 5 lasers (the Nd:YAG and excimer pump lasers, plus three dye lasers), and numerous new problems with beam diagnostics and spectroscopic constraints.

The system which we chose for these initial experiments was $I_{2}\left(X^{1} \Sigma_{q}\right)+H e$, with $\mathrm{I}_{2}$ prepared in initial states $v=5$ and $v=15$. Iodine was chosen not only for its spectroscopic convenience, but also because we have extensive data on excitation from the ground vibrational state with which to compare the excitedstate results. To incorporate SEP preparation of initial states, a window was added to the $\mathrm{I}_{2}$ source chamber so that two Lambda Physik $3002 \mathrm{E}$ dye lasers, synchronously pumped by a LPX-205i excimer laser at $308 \mathrm{~nm}$, could irradiate the seeded $\mathrm{I}_{2}$ beam in the space between the two collimating skimmers. A Molectron Nd:YAG-pumped dye laser was used to probe both the population of the SEP selected initial state prior to collision and the distribution of final product states after collision, by laser-induced fluorescence (LIF). In a typical experiment, about $10 \%$ of the gruund-state $\mathrm{I}_{2}$ population was transferred into the desired excited state.

Figure 1 shows LIF spectra of the primary beam with and without SEP to prepare the initial state $v=5$. As shown in the upper spectrum, nearly all of the primary beam population is initially in $v=0$. The lower spectrum shows the shift of population from $v=0$ to $v=5$ when the SEP is turned on. Note that the SEP process transfers population to $v=5$ only from those rotational states in $v=0$ 
which fall within t'ie laser bandwidth $(j=0-3)$, removing the long tail from the $\sim 1.5 \mathrm{~K}$ rotational distribution.

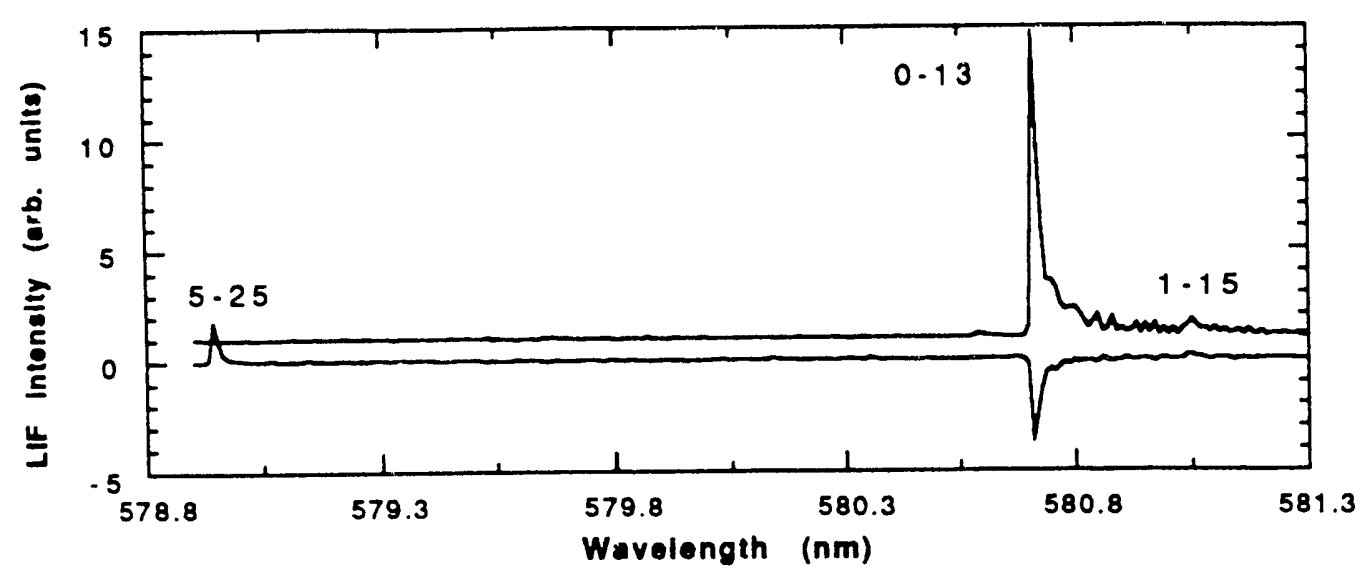

Figure 1. LIF spectra. The upper spectrum (displaced upward by one unit) shows the primary beam LIF spectrum with the SEP turned off. The lower spectrum is the difference (ON - OFF) with the SEP toggled on and off.

The collision energy was scanned over the range from $25 \mathrm{meV}$ to $196 \mathrm{meV}$ by varying the primary beam intersection angle. LIF signals for the selected final states were averaged over at least 25 scans, with 200 laser shots per point in each cycle. The resulting transition cross sections were normalized to the cross section for collisional attenuation of the initial state signal in the primary beam. As in the experiments with $v=0$ reported previously, the initial state attenuation becomes constant at energies higher than about $60 \mathrm{meV}$, indicating that essentially all strong collisions scatter molecules out of the small range of $j$ states detected at the bandhead. Thus, the attenuation cross section $\sigma_{a}$ is approximately equal to the gas-kinetic hard-sphere cross section of $42 \AA^{2}$. The results are shown in Figure 2 for the transitions $v=5 \rightarrow 4,6$ and $v=15 \rightarrow 16$. Also shown for comparison are the $v=0 \rightarrow 1$ risults from previous experiments. All of the transition cross sections for excited initial states are considerably larger than those for $v=0 \rightarrow 1$, even though all of the transitions involve transfer of only a single quantum oi nearly the same magnitude in each case $\left(\Delta E_{0,1}=213 \mathrm{~cm}^{-1}\right.$, $\Delta E_{5,6}=207 \mathrm{~cm}^{-1}$, and $\left.\Delta E_{15,16}=196 \mathrm{~cm}^{-1}\right)$. Furthermore, while the kinetic energy dependence of the $v=0 \rightarrow 1$ cross section is linear over this energy range, the transition cross sections for initially excited states are definitely not. The $v=15 \rightarrow 16$ cross section even displays a maximum at about $90 \mathrm{meV}$. 

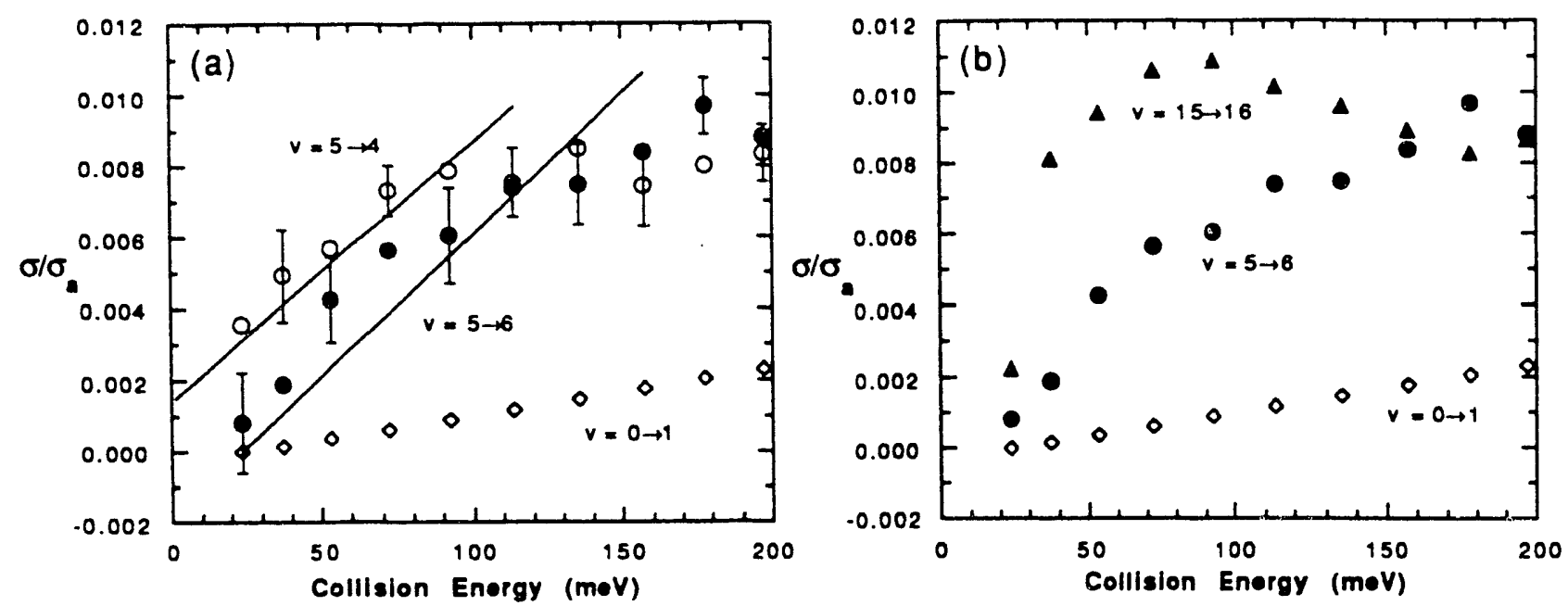

Figure 2. Kinetic energy dependence of the normalized transition cross sections $\sigma / \sigma_{a}$.

The first thing which one notices about these results is that there is a very strong dependence of cross sections for single-quantum transfer on the value of the initial quantum number. Since the magnitude of the energy quantum is nearly the same in each case, this effect has nothing to do with the "energy ge p laws" which have conventionally been used to scale rates or cross sections for different transitions. 1 However, while these results disprove the general applicability of scaling relationships based merely on the energy gaps for various transitions, they strongly suggest a much more fundamental scaling relationship based on the quantum numbers. These new scaling relations are derived most easily from the classical-quantal correspondence which exists for the forced harmonic oscillator problem, and which we have found extremely useful in the past for interpretation of a wide variety of vibrationally inelastic scattering data. ${ }^{2}$

When an force $F(t)$ (of arbitrary time dependence) acts on a quantum oscillator, the probability $P_{m, n}$ for the quantum vibrational transition $v=m \rightarrow n$

${ }^{1}$ For an excellent bibliography on energy gap laws, and their application to the interpretation of data on vibrational energy transfer in electronically excited $I_{2}$, see the work of Parmenter and coworkers: H. Du, D. J. Krajnovich and C. S. Parmenter, J. Phys. Chem. 95, 2104 (1991), and references cited therein.

2See W. R. Gentry, Ch. 12 in Atom-Molecule Collision Theory-a Guide for the Experimentalist, ed. by R. B. Bernstein (Plenum, New York, 1979), and references cited therein. 
can be expressed in terms of the energy $\Delta E$ transferred to an initially stationary classical oscillator by the identical force function. The relationship is:

where

$$
\begin{gathered}
P_{m, n}=\min _{\min (m, n)} e^{-\varepsilon} \varepsilon^{m+n} S_{m, n}^{2}, \\
S_{m, n}=\sum_{k=0} \frac{(-1)^{k} \varepsilon^{-k}}{(n-k) ! k !(m-k) !}, \\
\varepsilon=\Delta E / h v
\end{gathered}
$$

is the classical energy transfer in units of the quantum spacing. In order to apply this correspondence to the calculation of vibrationally inelastic cross sections, the transition probability must be averaged over initial conditions pertaining to the particular collision problem, such as impact parameter and orientation.

Our new scaling relationship, which will be described in detail in publication 17 in the above list, is based on an approximation to Eq. (1) which is valid in the limit of either small $\varepsilon$ or large quantum numbers $m, n$. The approximation is numerically quite good over a large range of $\varepsilon$ even for small $m, n$. In its most general form, the probability $P_{m, m+\Delta v}$ for the quantum vibrational transition $v=m \rightarrow m+\Delta v$ can be equated approximately to the probability $P_{0, \Delta v}$ for transition from the ground state, in the form

$$
P_{m, m+\Delta v}(\xi)=P_{0, \Delta v}(\varepsilon),
$$

where the quantum number scaling is all contained in the scaling of the classical energy transfer parameter

$$
\xi=\varepsilon\left[\frac{m ! \Delta v !}{(m+\Delta v) !}\right]^{\frac{1}{\Delta v}}
$$

(Here we assume that $\Delta v$ is positive. A similar relationship holds for relaxation channels with $\Delta v$ negative.)

In general, numerical calculations are required to calculate $\varepsilon$ (and therefore $\xi$ ) for a real system, and to average the resulting probabilities over impact parameter and orientation to obtain cruss sections. However, for nearly impulsive collisions such as $\mathrm{I}_{2}+\mathrm{He}$, we can use these scaling relationships directly, together with microreversibility, to predict the slopes of the cross sections 
near threshold for the transitions $v=5 \rightarrow 4,6$ and $v=15 \rightarrow 16$ from that measured for $v=0 \rightarrow 1$ (see publication 11 above). The results, shown as solid lines in Fig. 2(a), provide an adequate fit to the data at low energies.

We have also used this general classical-quantal correspondence approach to carry out a completely $a b$ initio calculation of the excitation cross sections as a function of kinetic energy and initial quantum number, in which the $\varepsilon$ values are calculated from classical trajectories on a realistic model potential energy surface. ${ }^{3}$ The results are shown in Fig. 3.

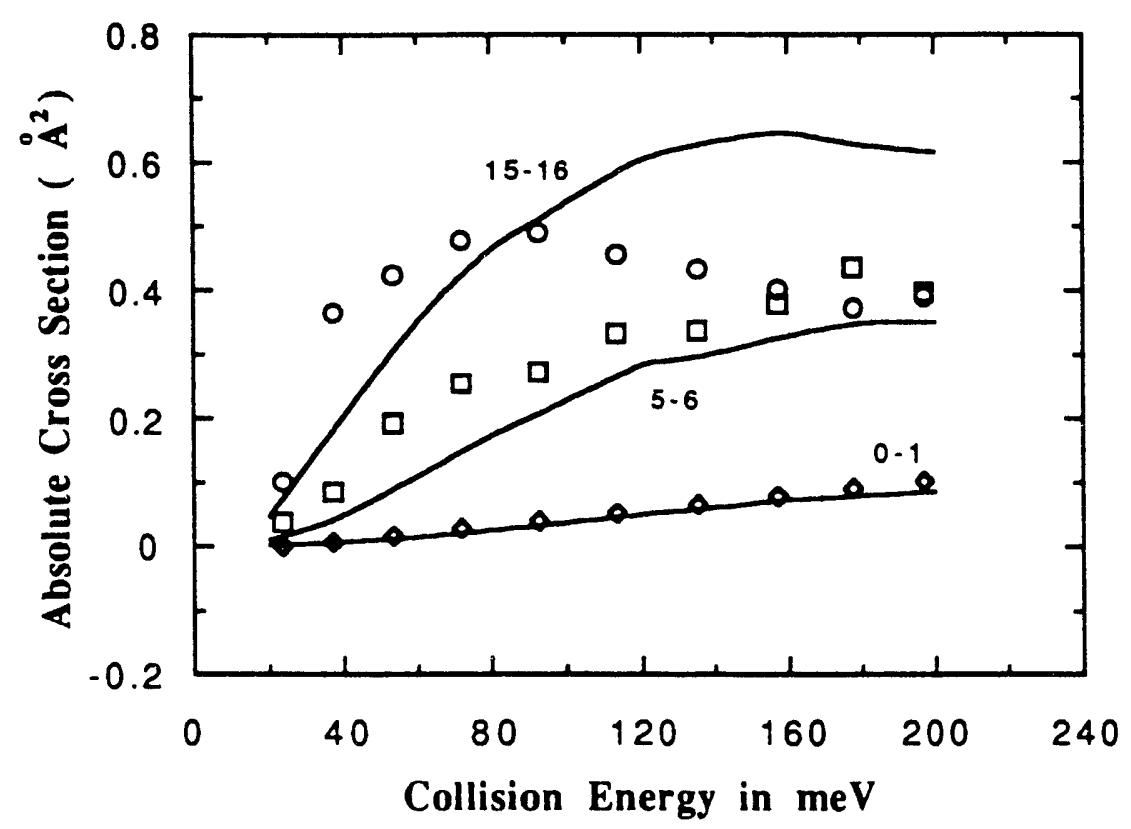

Figure 3. Comparison between experimental and theoretical cross sections for singlequantum excitation.

It is to be emphasized that there are no adjustable parameters in either the theoretical or experimental results given in Fig. 3. The absolute cross section comparisons require only that the experimental attenuation cross section be equated with the handbook value of $42 \AA^{2}$ from transport data. The agreement is rather remarkable given the neglect of anharmonic effects and the uncertain accuracy of the potential energy surface used for the calculations.

${ }^{3}$ D. W. Schwenke and D. G. Truhlar, Chem. Phys. Letters 98, 217 (1983). 
We believe that these results-particularly the large effects due to quantum number scaling-will have important implications for the modeling of energy transfer processes in complex systems such as flames.

\section{B. State-to-State Vibrational and Rotational Excitation of Glyoxal}

In our experiments with trans-glyoxal, just as in the experiments we carried out previously with aniline and para-difluorobenzene, we have continued to search for mode selectivity in the energy dependence of state-to-state microscopic cross sections. Our studies have been directed primarily toward understanding the details of pure rotational excitation and vibrational-rotational coupling in collisions of $\mathrm{S}_{0}$ trans-glyoxal with helium. To the best of our knowledge, these results represent the first inelastic scattering experiments for a molecule as large as glyoxal in which the $j$ and $k$ rotational energy levels are fully resolved, although results recently reported by Parmenter and coworkers ${ }^{4}$ do show $k$-resolved structure for collisions of $\mathrm{S}_{1}$ glyoxal.

Trans-glyoxal is an asymmetric molecule with rotational constants $A=1.844$ $\mathrm{cm}^{-1}, B=0.160 \mathrm{~cm}^{-1}$, and $C=0.147 \mathrm{~cm}^{-1}$. Because $B$ and $C$ are so nearly equal, it can be accurately treated as a prolate symmetric top, with total and projection angular momentum quantum numbers $j$ and $k$, respectively. Due to the large difference between $A$ and $B$, transitions corresponding to values of $j, k$ crowd together to form $k$ sub-bands in the rotational spectrum. The $\mathrm{r} R$ branch of the laser-induced fluorescence (LIF) spectrum was resolved in $k$ using a gratinglimited laser bandwidth of $0.7 \mathrm{~cm}^{-1} \mathrm{fwhm}$, and was further resolved in $j$ with the aid of a pressure-tuned etalon, yielding a bandwidth of $0.04 \mathrm{~cm}^{-1} \mathrm{fwhm}$. Fig. 4 shows the primary beam and the collision-induced difference spectra for the $0_{0}$ band of the electronic transition. A least squares fit revealed the initial state distribution in the beam to be approximately described by two temperatures, with $95 \%$ of the population at $1.5^{\circ} \mathrm{K}$ and $5 \%$ of the population at $30^{\circ} \mathrm{K}$. The collisioninduced difference spectrum clearly shows the population transfer from low $k$ to high $k$.

\footnotetext{
${ }^{4}$ K. W. Butz, H. Du, D. J. Krajnovich and C. S. Parmenter, J. Chem. Phys. 89, 4680 (1988).
} 
For collisions of trans-glyoxal and helium, our crossed molecular beam apparatus with variable beam intersection angle permits the center-of-mass collisics energy to be varied between about $150 \mathrm{~cm}^{-1}$ and $1400 \mathrm{~cm}^{-1}$, with an energy spread of about $10 \%$. A search for vibrational excitation was carried out for the lowest frequency modes $7,12,5,8$, and 4, having frequencies between 127 and $1065 \mathrm{~cm}^{-1} .5$ The only mode discovered to be excited with measurable probability was the lowest-frequency mode 7 , which corresponds to torsion about the C-C axis. The primary beam and collision-induced difference spectra in the region of the $71^{1}$ transition are shown in Fig. 5. The blue tail of this spectrum shows obvious $k$ excitation. Of the $7 \%$ of the molecules in the beam which collide

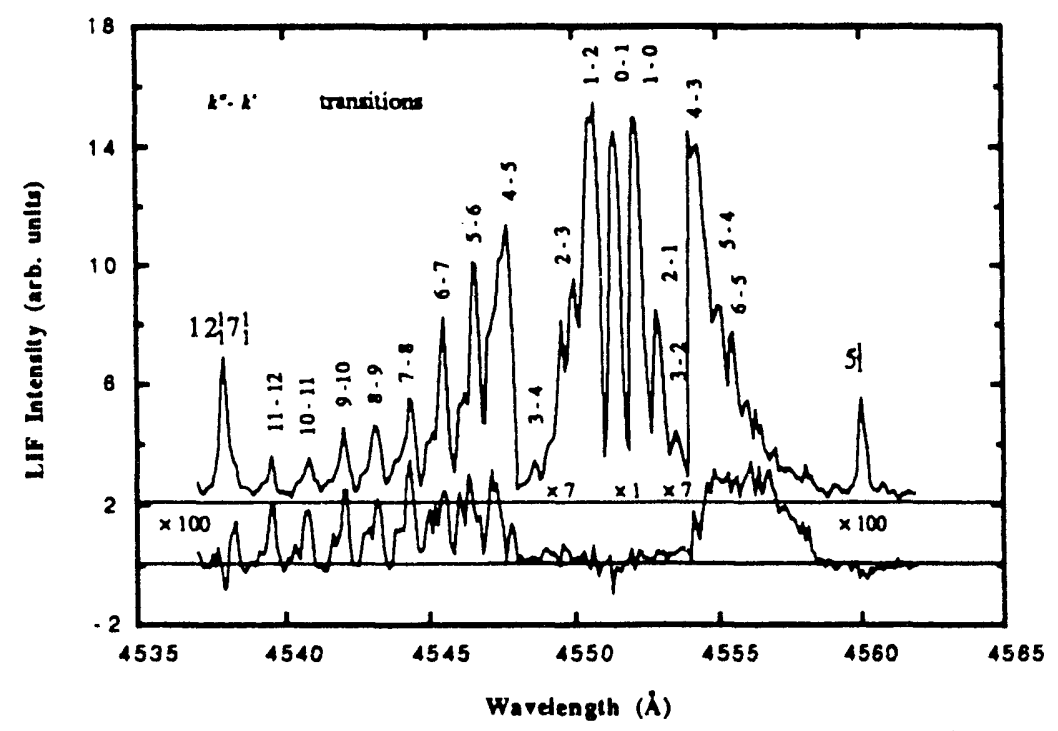

Figure 4. LIF spectrum showing $k$ rotational structure within the ground vibrational state, at a collision energy of $374 \mathrm{~cm}^{-1}$. The He off signal is shown above, and the collision-induced difference signal below. Attenuation of low $k$ and population of high $k$ can be seen. The difference spectrum shows no excitation of mode 5 .

${ }^{5}$ G.H. Atkinson, R.A. Malstrom, and M.E. McDwain, J. Mol. Spectry. 76, 164 (1979). 


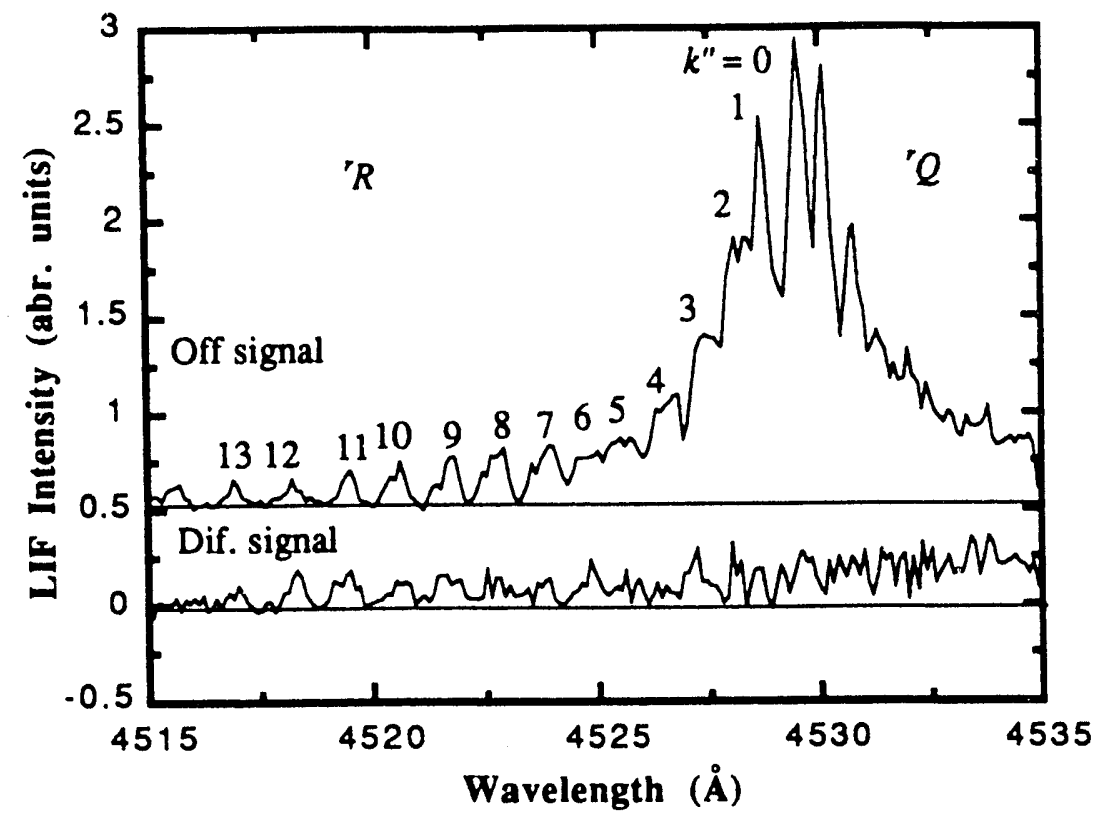

Figure 5. LIF spectrum showing $k$ rotational structure within vibrational mode 7 , at a collision energy of $374 \mathrm{~cm}^{-1}$. The difference signal shows excitation of the $k$ structure.

with $\mathrm{He}$, about $10 \%$ are excited to $7_{1}$ and less than $1 \%$ to $7_{2}$ at $374 \mathrm{~cm}^{-1}$ collision energy. Almost all of the remainder undergo pure rotationally inelastic scattering. At a collision energy of $1400 \mathrm{~cm}^{-1}$, about $25 \%$ are excited to $7_{1}$. This behavior is similar to that observed previously for $\mathrm{S}_{0}$ aniline and $\mathrm{pDFB}$, in which the low-frequency modes were also found to be especially active, and with that seen by Parmenter and coworke's for $\mathrm{S}_{\mathfrak{I}}$ trans-glyoxal. However, the $\mathrm{S}_{0}$ transglyoxal energy transfer dynamics are markedly different from those of pDFB in another respect. Mode 7 vibrational excitation in trans-glyoxal is accompanied by large amounts of rotational excitation, comparable to that which is observed in the vibrationally elastic scattering.

This large degree of vibrational-rotational coupling is probably due to the fact that the torsional motion in mode 7 is geometrically similar to rotation about the principal axis in trans-glyoxal, the difference being only whether the two CHO moieties are rotating in the same direction (rotation) or opposite directions (vibration). A search for excitation of other vibrational modes was carried out with $\mathrm{Ne}$ instead of $\mathrm{He}$ as a collision partner, but again excitation of only mode 7 was observed. 


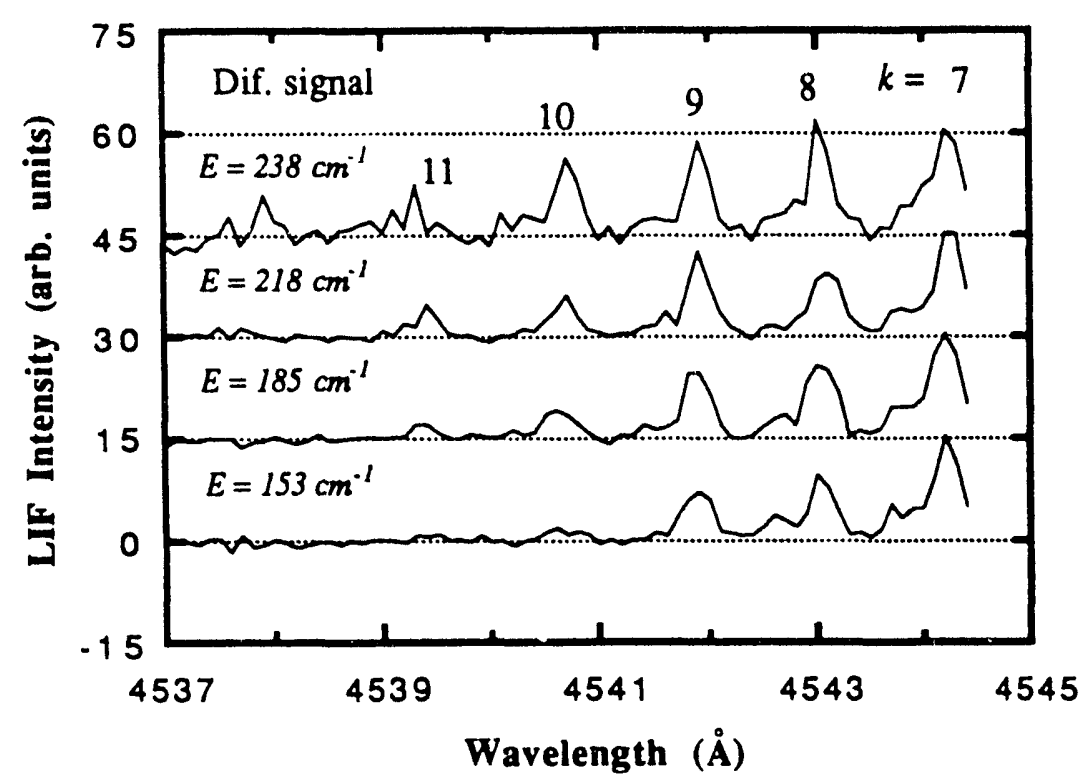

Figure 6. LIF spectra showing $k$ structure within the ground vibrational state, as a function of collision energy.

We also examined thoroughly the extensive rotational excitation within the vibrational ground state. As illustrated in Fig. 6, at all collision energies within the experimental range the net transfer of population by collision was negative for $k=0$ and positive for all values of $k$ higher than $k=1$. Ai a kinetic energy of 1400 $\mathrm{cm}^{-1}$, the excitation extends almost evenly to at least $r=12$. At lower collision energies, the maximum values of $k$ which were appreciably populated were $k=9$ at $153 \mathrm{~cm}^{-1}, k=10$ at $185 \mathrm{~cm}^{-1,} k=11$ at $218 \mathrm{~cm}^{-1}$ and $k=12$ at $238 \mathrm{~cm}^{-1}$. In each case, the excitation goes out essentially to the energetic limit. The threshold and saturation behaviors were also observed directly by monitoring the population in $k=10, j=10$ as a function of collision energy. All these results are consistent with a very large anisotropy in the interactiol: potential.

For vibrationally elastic scattering, we carried out high resolution $j, k$ resolved measurements for $k=0, j \leq 7, k=1, j \leq 8, k=3 j \leq 11, k=5 j \leq 14, k=7, j \leq 12$, and $k=9, j \leq 14$. Taking into account rotational degeneracies, nuclear spin statistics and Hönl-London factors, we determined a branching ratio for each $j, k$ state. It should be noted that the relative pcpulations of individual $j$ states for a common value of $k$ are better determined than are the relative populations for different $k$ states, because the spectrum for each value of $k$ was measured in a separate experiment. For $k=0$ and $k=1$, especially at low $j$, the net transfer of population is 
negative. For values of $K \geq 5$, the net population transfer is positive, with a maximum for each $k$ at $j=k+1$.

We had previously discovered that a simple rigid-shell double-ellipsoid model was capable of reproducing the principal features of the pure rotational excitation in pDFB $+\mathrm{He}$ collisions, and therefore expected that a similar treatment might be appropriate for glyoxal. However, glyoxal is not even approximately ellipsoidal in shape, so we developed a new model for the repulsive potential shape based on the superposition of hard spheres centered on each atom, each having the appropriate van der Waals radius. A classical Monte Carlo calculation was then carried out to predict the final distribution of $j, k$ states from the initial distribution determined experimentally.

Fig. 7 shows the comparison. In general, the simple model reproduces the major features mentioned above, including the maximum in excitation at $j=k+1$. A single adjustable parameter was used to scale the experimental data to the model calculations, resulting in cross section magnitudes which are remarkably consistent with the theoretical model for all values of $k$. The main differences between theory and experiment are seen in the $k=0$ and $k=1$ simulations, where the predicted populations in high $j$ are too small, and in $k=3$, where the theory predicts substantial negative population transfer from high $j$, which is not observed experimentally. Refinements of the calculations are under way to whether these discrepancies can be reduced by appropriate modifications to the potential. Preliminary results indicate that the radius assumed for the carbon atom in the original calculations was too small. On the whole, however, this seems to be an excellent way to approach rotational energy transfer in polyatomics. 


$$
k=0
$$

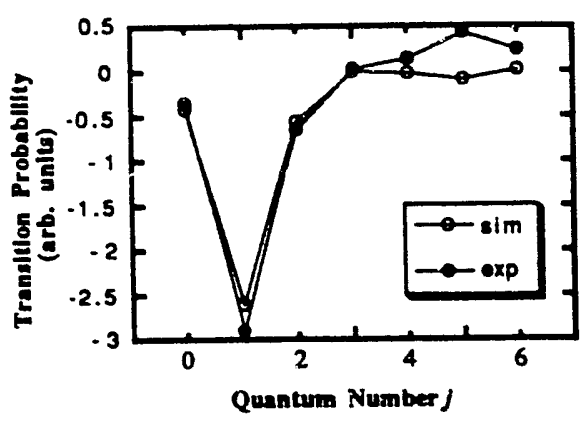

$$
k=3
$$

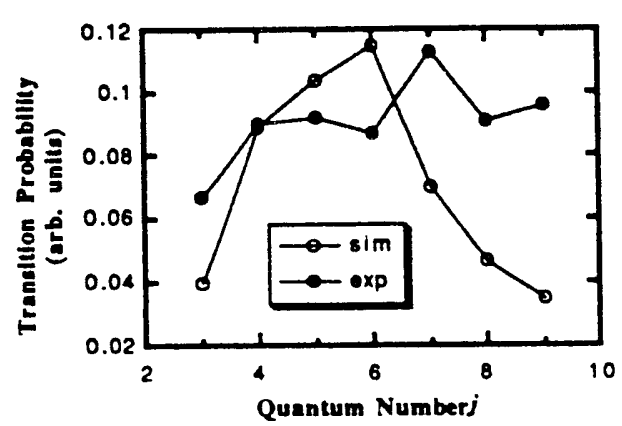

$$
k=7
$$

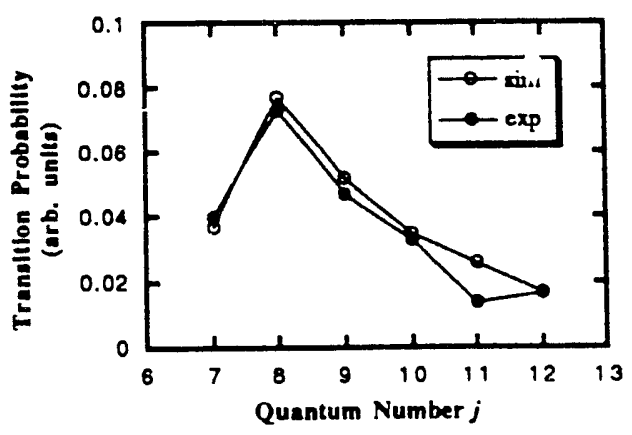

$k=1$

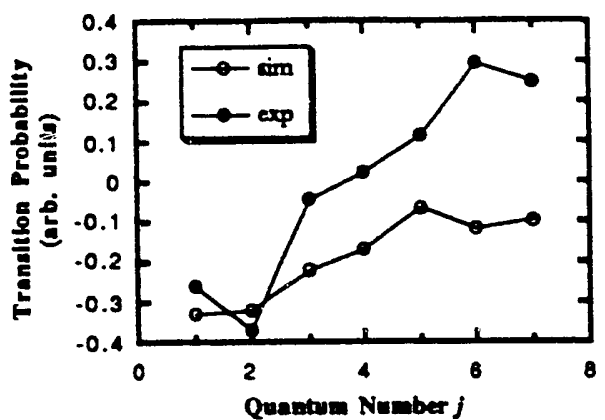

$k=5$

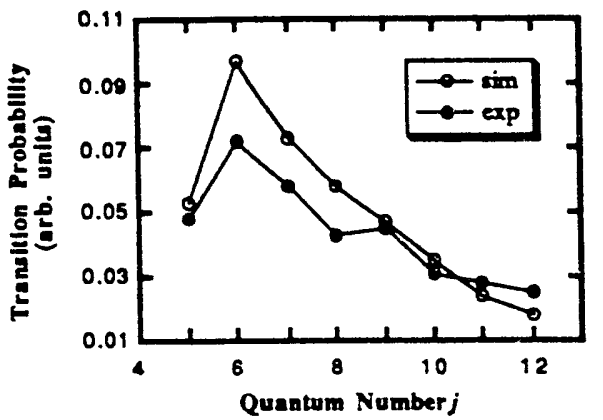

$k=9$

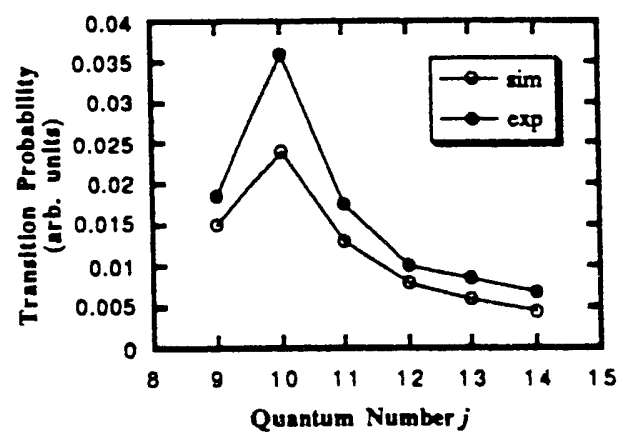

Figure 7. Comparison of experimental and theoretical $j, k$ distributions following collision. In each plot, the distribution of $j$ is shown for the $k$ value indicated above. 


\section{Fotational Excitation of $\mathbf{I}_{2}$}

Our early experiments on $\mathrm{I}_{2}$ vibrational excitation, together with the calculations of Schwenke and Truhlar ${ }^{\circ}$ on $\mathrm{He}+\mathrm{I}_{2}$, showed a high sensitivity of the rotationally-averaged vibrational excitation cross sections to details of the rotational state distribution, despite the fact that the rotational excitation which accompanies vibrational excitation is quite small (most-probable $\Delta j \approx 6$ ). Indeed, rotational excitation is clear', the dominant process in the collision dynamics. Virtually every collision is rotationally inelastic, while typically only $10^{-3}$ or less of the collisions are vibrationally inelastic. Thus, the angular anisotropy of the force component driving the vibrational coordinate is relatively small, while the anisotropy of the full interaction potential is large. Clearly, all inelastic channels must be considered together to provide a good description of the energy transfer dynamics. In our first experiments with our new higher-resolution laser, we therefore carried out a comprehensive investigation of the pure rotational excitation in $\mathrm{I}_{2}+\mathrm{He}$ collisions, to complement our previous results on rovibrational excitation. In the LIF spectra taken with the etalon-narrowed laser, individual $j$ states are resolved for $j>4$.

We measured both the kinetic energy dependence for exciting a specific final rotational state, and the distribution of final rotational states at a given collision energy. Mlustrations of both types of experimental result are given in Figs. 8 and 9.

${ }^{6}$ D. W. Schwenke and D. G. Truhlar, J. Chem. Phys. 81, 5586 (1984). 


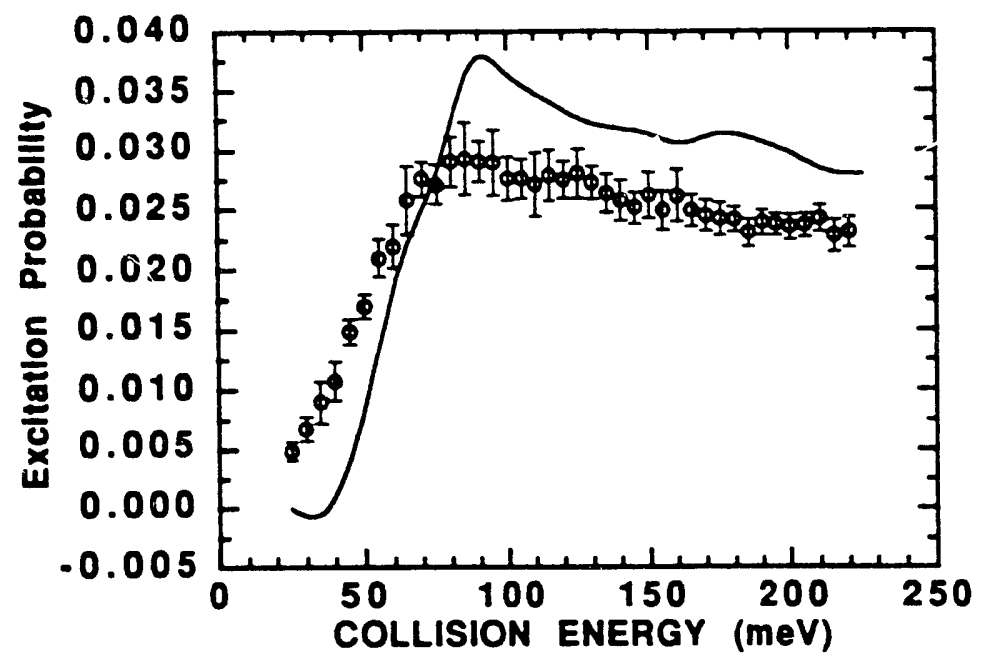

Figure 8. Cross section for exciting $j=30$ as a function of the kinetic energy of collision. The excitation probability shown is the ratio of the excitation cross section to the (constant) attenuation cross section. Also shown for comparison (solid line) is the result of a classical rigid-ellipsoic calculation.

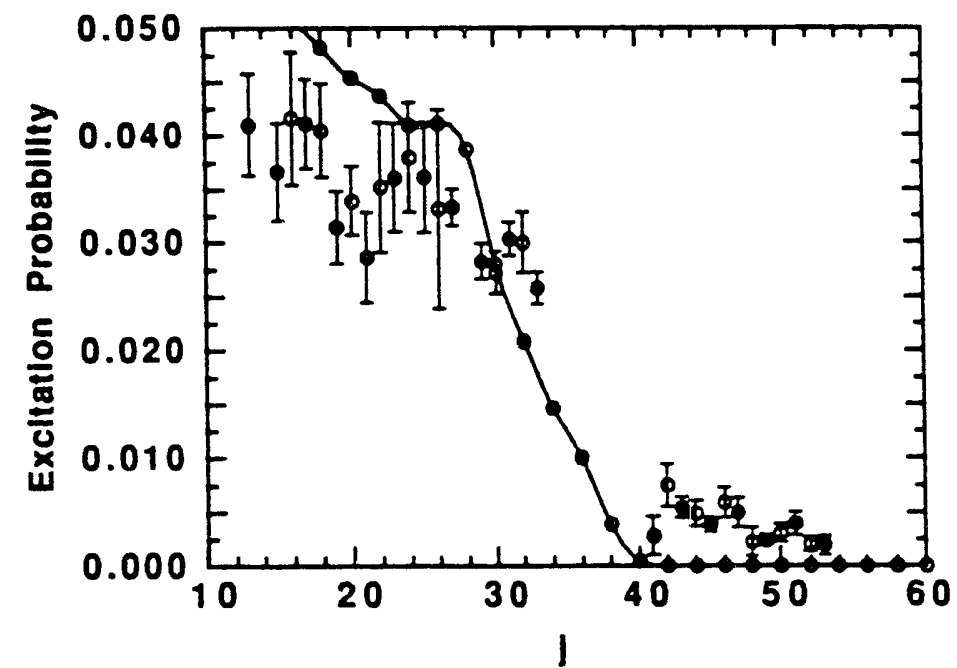

Figure 9. Cross section as a function of the final $j$ state for excitation from the initial manifold of states at the beam temperature of $1.5 \mathrm{~K}$. The solid line is the result of a classical rigid-ellipsoid calculation.

Both types of data show clear rotational rainbow structure which is directly related to the anisotropy of the interaction potential. ${ }^{7}$ This is exhibited in the

\footnotetext{
${ }^{7}$ W. Schepper, U. Ross, D. Beck, Z. Phys. A 290, 131 (1979); D. Beck, U. Ross and W. Schepper, Phys. Rec A 19, 2173 (1979); Verh. Dtsch. Phys. Ges. 5, 533 (1980).
} 
dynamic threshold behavior of the cross section for exciting a specific high rotational state (Fig. 8), and in the cutoff of the cross section as a function of final rotational state at a specific collision energy (Fig. 9). It has proven to be possible to model these rotationally inelastic collisions quite successfully using a simple rigid-ellipsoid potential having an anisotropy parameter (difference between the major and minor radii of the ellipsoid) of $1.8 \AA$. The comparisons shown in Figs. 8 and 9 show only small deviations from the data. These aic attributable to quantum effects which tend to blur the classical rainbow singularities. ${ }^{8}$ The vibrational and rotational energy transfer in $\mathrm{I}_{2}+\mathrm{He}$ collisions can now be said to be understood virtually completely, apart from minor adjustments in the interaction potential which will be necessary to achieve quantitative agreement between theory and experiment with respect to the vibrational-rotational coupling. ${ }^{9}$

\section{Rotational Excitation of p-Difluorobenzene}

A great deal of effort in our laboratory has been directed toward understanding the details of pure rotational excitation and vibrational-rotational coupling in collisions of pDFB with He. Because of the spectroscopic complexity of pDFB, this problem has proven to be very difficult. Nevertheless, we believe that we now have a pretty clear qualitative picture of the energy transfer dynamics, at least within the ground vibrational state and the $30_{1}$ excited state. Our previous vibrational excitation experiments on this system ${ }^{10}$ were carried out with an effective spectroscopic resolution of about $0.7 \mathrm{~cm}^{-1}$, which was adequate to characterize mode selectivity in the product vibrational state distribution. We have now carried out LIF detection of the scattered products with a frequencydoubled bandwidth of about $0.05 \mathrm{~cm}^{-1}$, which has made it possible to observe a great deal of the rotational structure as well.

The pDFB molecule is an asymmetric top, although it is close enough to a prolate symmetric top to permit the symmetric top description to be applied accurately to high- $j$ states and to permit deviations from a symmetric top

8R. Schinke, J. Chem. Phys. 75, 5449 (1981).

${ }^{9}$ M. J. Unekis, D. W. Schwenke and D. G. Truhlar, unpublished results.

${ }^{10}$ G. Hall, C. F. Giese and W. R. Gentry, J. Chem. Phys. 83, 5343 (1985). 
description to be modeled with small perturbations at low $j$. Each rotational level in the ground electronic state connects with 6 bands, in the upper resonance electronic state, which, in the symmetric top notation, can be labelled ${ }^{\mathrm{r}} \mathrm{P},{ }^{\mathrm{r}} \mathrm{Q},{ }^{\mathrm{r}} \mathrm{R}$ and ${ }^{P P}, P Q, P R$. Since $j$ and $k$ span at least the range 0 to 30 in these experiments, the individual $(\Delta k, \Delta j)$ transitions number in many thousands. We are therefore not able to resolve individual $j, k$ states. It has, however, proven to be possible to assign certain features of the observed spectra with confidence, and therefore to draw some definite, although qualitative, conclusions about the dynamics.

An expanded view of the blue-wing region is shown in Fig. 10. The low-j region of the $22_{1}^{1}$ hot band, seen in the primary beam spectrum at $4.35 \mathrm{~cm}^{-1}$, is attenuated in the difference spectrum because of rotational excitation, in the same way that the $0_{0}$ band is attenuated. The interesting foatures of the collisioninduced spectrum are peaks which closely match calculated ' $R$ branch bandheads for $j$ between about 12 and 20 . An analysis based on rigid-rotor calculations shows that each $j$ state gives rise to a band in which the $k$-state progression has a turning point at $k \approx 10$, forming a bandhead in which the frequencies for $k \approx 6$ to 13 are nearly coincident. The calculated bandhead frequencies, shown at the top of Fig. 10, are in good agreement with the observed structure. The small discrepancies observable for large $j$ are probably due to centrifugal distortion. On the basis of the observed intensity distribution in this region, we conclude that the most-probable final value of $j$ arising from $\mathrm{He}$ collisions at $605 \mathrm{~cm}^{-1}$ kinetic energy is $j_{m p} \approx 16$. 


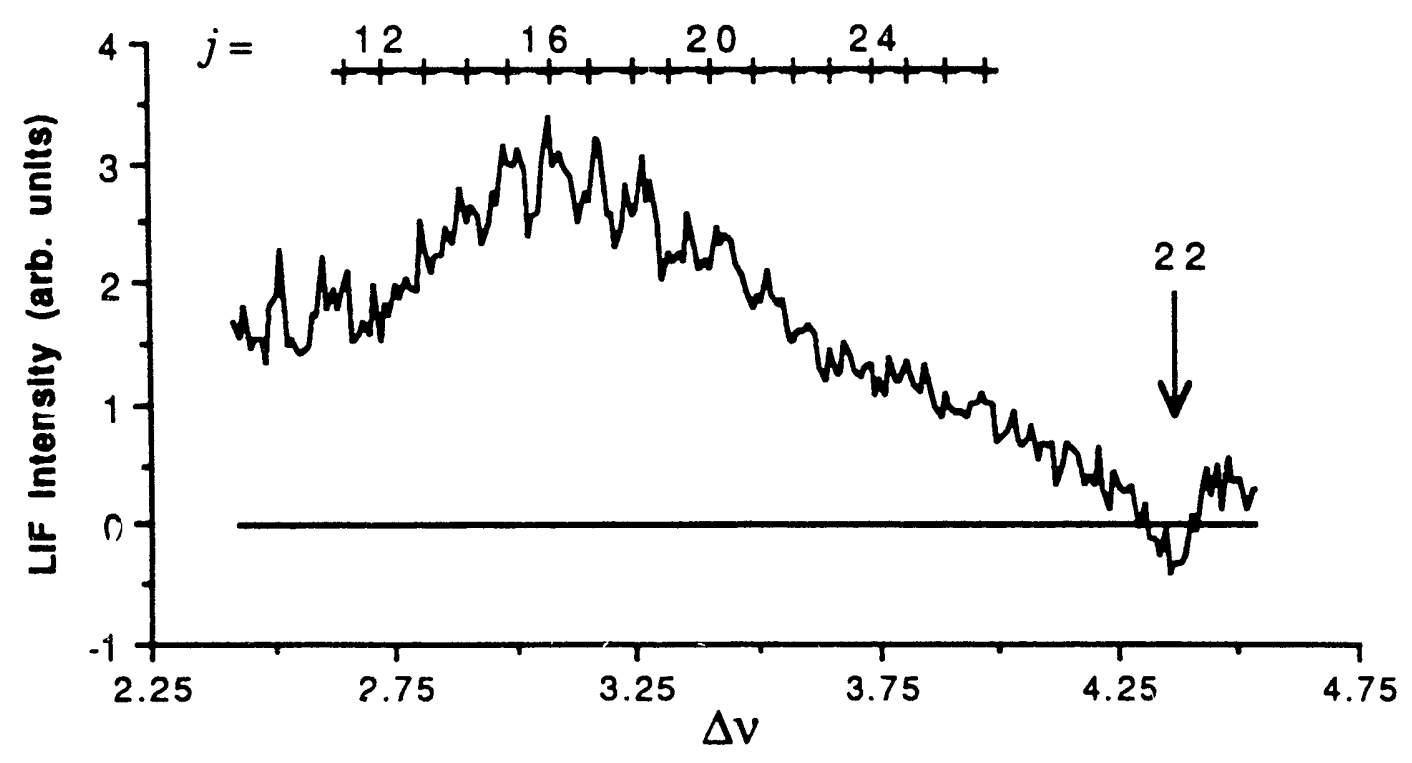

Figure 10. Collision-induced LIF difference spectrum in the blue wing of the $O_{0}$ band. The collision energy is $135 \mathrm{meV}$. Bandheads for various $j$ values are partially resolved, showing a broad distribution with a most-probable value $j=16$.

The red-wing spectral region, shown in Fig. 11, contains information on the $k$-state distribution which is conveniently complementary to that provided by the blue-wing data on the $j$-state distribution. In the ${ }^{P Q}$ branch, each $k$ state gives rise to a band in which all $j$ states are nearly coincident in frequency. The difference spectrum shows the resulting collision-induced distribution of $k$ quite clearly, including the even/odd intensity ratio of 5/3 expected from the nuclear spin statistics. The distribution of $k$ is evidently quite broad, but with a mostprobable value which we can estimate as $k_{m p} \approx 8$. The energy of $(j, k)=(16,8)$ is only $21 \mathrm{~cm}^{-1}$, or $3.5 \%$ of the collision energy. Interestingly, this is almost the same most-probable final rotational energy as was observed for $\mathrm{I}_{2}+\mathrm{He}$ scattering at the same collision energy, while the rotational rainbow cutoff in that case was observed to be at about $10 \%$ of the collision energy. 


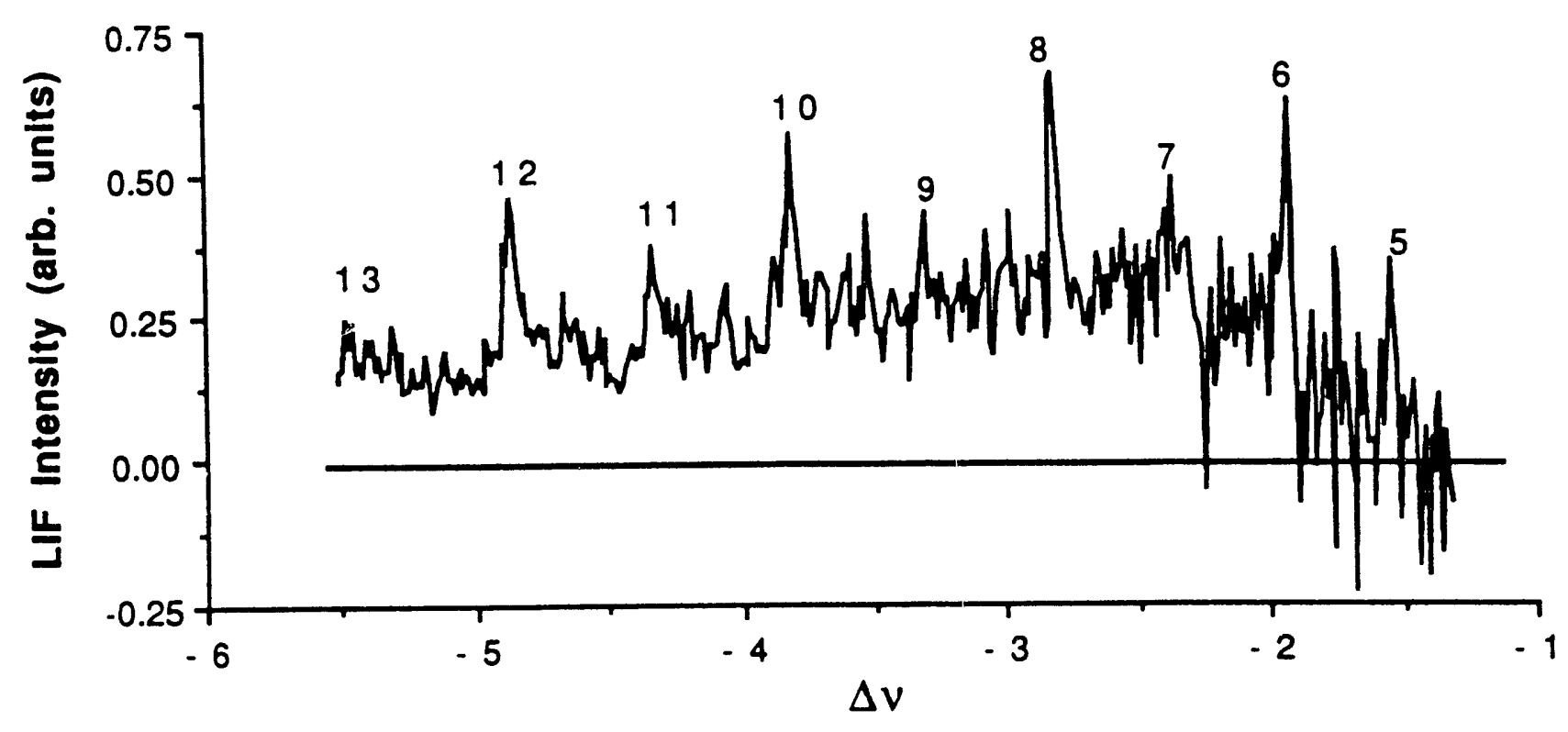

Figure 11. Collision-induced LIF difference spectrum in the red wing of the $0_{0}$ band. Bandheads are labeled with values of the $k$ quantum numbers.

We have also carried out classical trajectory calculations on the $\mathrm{pDFB}+\mathrm{He}$ system, modeling the interaction potential as a rigid-shell double-ellipsoid based on handbook values of the atomic radii (with no adjustable parameters). Remarkably, the calculations show broad distributions of $j$ and $k$, with mostprobable values of 16 and 8 , respectively.

Finally, we must comment on the vibrational-rotational coupling evident in the LIF spectra for collision-induced population of the $30_{1}^{1}$ band. Mode 30 is the only vibrational mode, out of many which are accessible energetically, which is excited with measurable probability. It was our hope that information on the $(j, k)$ distribution of rotational states accompanying vibrational excitation would provide insights into what collision geometries lead preferentially to vibrational excitation. Since pDFB is approximately a prolate top having a symmetry axis through the two fluorine atoms, $k$ represents the projection of $j$ on the F-F axis. For purposes of illustration, we can distinguish qualitatively among several limiting cases, characterized by whether the impact parameter $b$ is small or large, and if $b$ is large whether collision is with the $\mathrm{F}$ atom ends of the molecule or with the $\mathrm{CH}$ sides of tia molecule. If $b$ is small, both $j$ and $k$ will be small whether 
the collision is with the center or the side of the ring. If $b$ is large, $j$ will also generally be large, but collisions with an $\mathrm{F}$ atom will led to small $k$, while collisions with the side of the ring will lead to large $k$. Thus, the $j, k$ distribution within an excited vibrational state should be related directly to the distribution of collision geometries in the special subset of collisions which induce vibrational excitation.

The remarkable thing about the $30_{1}^{1}$ rotational band contour in the collisioninduced difference spectrum is that, unlike the $0_{0}$ band, it is very similar in both shape and width to that of the rotationally cold primary beam. The clear conclusion is that rotational excitation is much less efficient in those collisions which excite $30_{1}$ than in collisions which do not induce vibrational excitation. Our interpretation is that mode 30 excitation occurs primarily in small impact parameter collisions, in which the $\mathrm{He}$ atom strikes the benzene ring near its center and exerts little torque about any of the three axes. These collision geometries comprise only a small subset of the total. 

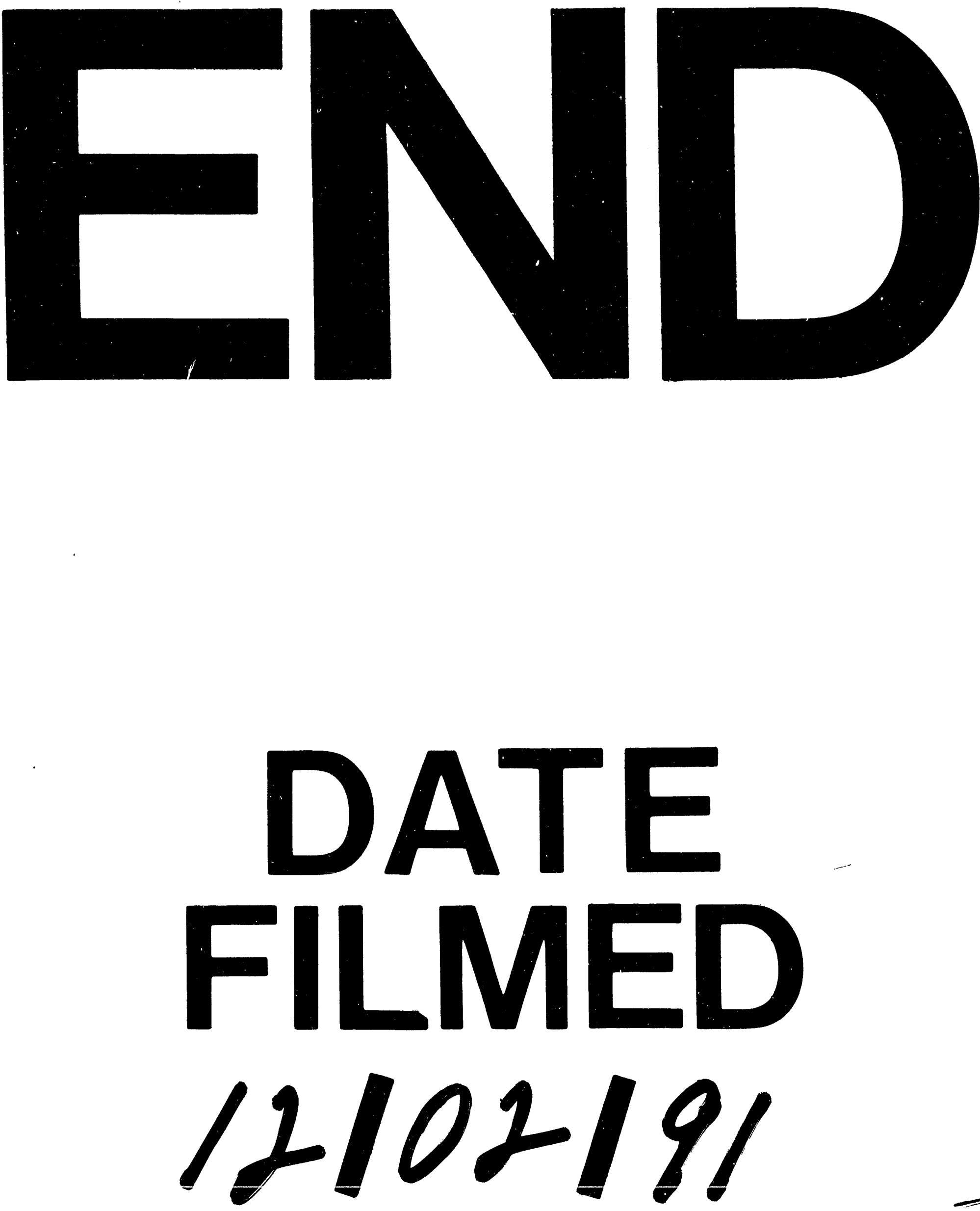

1 
